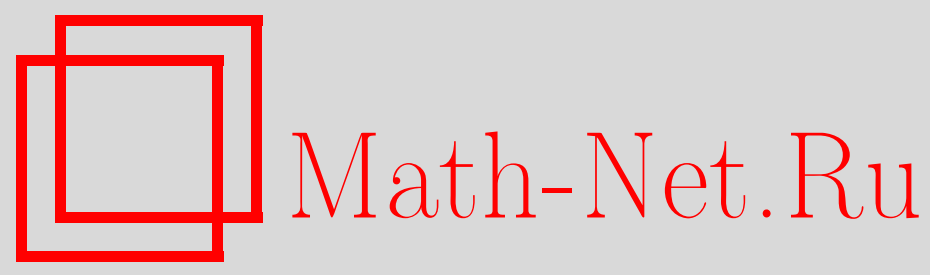

В. Н. Дубинин, О полиномах с критическими значениями на отрезке, Матем. заметки, 2005, том 78, выпуск 6, 827832

DOI: https://doi.org/10.4213/mzm2653

Использование Общероссийского математического портала Math-Net.Ru подразумевает, что вы прочитали и согласны с пользовательским соглашением http://www.mathnet.ru/rus/agreement

Параметры загрузки:

IP : 18.207 .199 .55

26 апреля 2023 г., 16:28:57

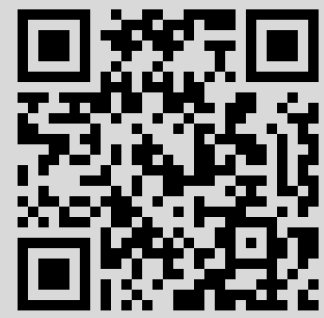




\section{О ПОЛИНОМАХ \\ С КРИТИЧЕСКИМИ ЗНАЧЕНИЯМИ НА ОТРЕЗКЕ}

\section{В.Н. Дубинин}

Для полиномов $P(z)$ с вещественньми коэффициентами и с фиксированным старшим коэффициентом, удовлетворяющих условиям $P(z) \in[-1,1]$ при $z \in[-1,1]$ и $P(z) \in[-1,1]$, когда $P^{\prime}(z)=0$, получены новые теоремы покрытия, неравенство бернштейновского типа и неравенства для коэффициентов. Доказательства основаны на применении конформного и однолистного отображения.

Библиограффия: 9 названий.

1. Введение. Пусть $P(z)=a z^{n}+\cdots-$ алгебраический полином степени $n$ с вещественными коэффициентами, удовлетворяющий условию

$$
|P(z)| \leqslant 1 \quad \text { для } z \in[-1,1] .
$$

Многочис ленные свойства полиномов с такой нормировкой восходят к классическим работам Чебышева, Маркова, Бернштейна и активно исследуются вплоть до настоящего времени (см., например, [1]-[3]). Часть этих свойств содержит информацию о некоторых коэффициентах полинома $P(z)$. Так, в недавней работе [4] помимо других результатов получены точные неравенства бернштейновского типа для указанных вьше полиномов с участием старшего коэффициента $a$. Знаки равенства при этом достигаются в случае полинома Чебьшева первого рода $T_{n}(z)=2^{n-1} z^{n}+\cdots$, т.е. в случае, когда $a=2^{n-1}$. Хорошо известно, что при условии (1) вьполяется неравенство $0<|a| \leqslant 2^{n-1}$ с равенством только для $P(z)= \pm T_{n}(z)$. Естественно поставить вопрос о получении аналогичных [4] свойств полиномов, где бы экстремальным оказался полином с наперед заданным старшим коэффициентом. Внимание автора к этой проблеме было инициировано В.И. Буренковым на Международной школе-конференции по анализу и геометрии, посвященной 75-летию Ю. Г. Решетняка (Новосибирск, 2004). В данной заметке указанная задача решается для полиномов, чьи значения в критических точках принадлежат отрезку $[-1,1]$. Для фиксированных $a, 0<a<2^{n-1}$, и $n \geqslant 2$ обозначим через $\mathscr{P}_{n}^{a}$ совокупность всех полиномов $P(z)=a z^{n}+\cdots$ степени $n$ с вещественньми коэффициентами, удовлетворяющих условию (1) и таких, что если в некоторой

Работа выполнена при финансовой поддержке ДВО РАН, грант № 05-III-A-01-039, и программы “Университеты России”, грант № 04.01.053. 
точке $z$ вьполняется $P^{\prime}(z)=0$, то необходимо $P(z) \in[-1,1]$. Ниже будут доказаны теоремы покрытия, неравенство бернштейновского типа и неравенства для коэффициентов полиномов класса $\mathscr{P}_{n}^{a}$. Экстремальньгм в каждом случае будет полином

$$
T_{n}^{a}(z):=T_{n}(b z+b-1)=a z^{n}+\cdots,
$$

где $b=\left(a 2^{1-n}\right)^{1 / n}<1$. Напомним представление полинома Чебьшева $T_{n}(z)$ через конформные отображения. Пусть

$$
\eta=\Phi(\omega)=\omega+\sqrt{\omega^{2}-1}
$$

означает ту ветвь аналитической функции, обратной функции Жуковского, которая конформно и однолистно отображает внешность отрезка $[-1,1]$ на круг $|\eta|<1$. Тогда

$$
T_{n}(z)=\frac{1}{2}\left(\Phi^{n}(z)+\Phi^{-n}(z)\right), \quad z \in \mathbb{C} .
$$

Ключевую роль в приведенных ниже доказательствах играет функция

$$
w=g(\zeta):=\left[\Phi\left(P\left(\frac{1}{2}\left(\zeta+\frac{1}{\zeta}\right)\right)\right)\right]^{1 / n}=\frac{1}{b} \zeta+\cdots,
$$

которая согласно лемме 2.7 работы [4] и ввиду (1) конформно и однолистно отображает область $\{\zeta:|\zeta|<1, P((\zeta+1 / \zeta) / 2) \notin[-1,1]\}$ на круг $|w|<1$. Здесь $P(z) \in \mathscr{P}_{n}^{a}$ и $1^{1 / n}=1$. Соответственно обратная $w=g(\zeta)$ функция

$$
\zeta=h(w)=\left(a 2^{1-n}\right)^{1 / n} w+\cdots
$$

принадлежит классу ограниченных однолистных в круге $|w|<1$ функций, экстремальные свойства которых хорошо изучены в литературе (см., например, [5]).

2. Теоремы покрытия. Обозначим через $\zeta=p(w ; b)$ функцию Пика, заданную соотношениями

$$
\frac{\zeta}{(1+\zeta)^{2}}=\frac{b w}{(1+w)^{2}}, \quad|w|<1, \quad|\zeta|<1, \quad \zeta \notin[d, 1)
$$

где $d=2 b^{-1}-1-2 \sqrt{b^{-1}\left(b^{-1}-1\right)}, 0<b<1$.

Следуя методике работы [4], приходим к теоремам покрытия образов и прообразов эллипсов при отображении полиномами $P(z) \in \mathscr{P}_{n}^{a}$.

Теорема 1. Для любого полинома $P(z)$ класса $\mathscr{P}_{n}^{a}$ и любого числа $\rho, 0<\rho<1$, прообраз әллипса с фокусами в точках \pm 1 и большой полуосъю $(\rho+1 / \rho) / 2$ лежит в кольчевой области $G(n, a, \rho)$, ограниченной двумя әллипсами с фокусами в точках \pm 1 и большими полуосями, равными

$$
\frac{1}{2}\left(-p\left(-\rho^{1 / n} ; b\right)-\frac{1}{p\left(-\rho^{1 / n} ; b\right)}\right) \quad u \quad \frac{1}{2}\left(p\left(\rho^{1 / n} ; b\right)+\frac{1}{p\left(\rho^{1 / n} ; b\right)}\right),
$$

где $b=\left(a 2^{1-n}\right)^{1 / n}$. Исключение составляет случай, когда $P(z) \equiv T_{n}^{a}(z)$ и, следовательно, прообраз указанного әллипса есть әллипс, все точки которого принадлехат области $G(n, a, \rho)$, кроме точек

$$
\frac{1}{2}\left(p\left(-\rho^{1 / n} ; b\right)+\frac{1}{p\left(-\rho^{1 / n} ; b\right)}\right) \quad u \quad \frac{1}{2}\left(p\left(\rho^{1 / n} ; b\right)+\frac{1}{p\left(\rho^{1 / n} ; b\right)}\right),
$$

которые лежсат на ее границе. 
ДокАЗАТЕЛЬСтво. По заданному полиному $P(z)$ класса $\mathscr{P}_{n}^{a}$ построим функцию $w=g(\zeta)(2)$ и рассмотрим обратное отображение $\zeta=h(w)=b w+\cdots$. Для любой точки $w$ на окружности $|w|=\rho^{1 / n}$ справедливы неравенства Пика [5, c. 55]

$$
p\left(\rho^{1 / n} ; b\right) \leqslant|h(w)| \leqslant-p\left(-\rho^{1 / n} ; b\right) .
$$

Это означает, что точка $z=(\zeta+1 / \zeta) / 2, \zeta=h(w)$, принадлежит замьканию области $G(n, a, \rho)$, в то время как точка $P(z)=\left(w^{n}+1 / w^{n}\right) / 2$ является произвольной точкой эллипса с фокусами в точках \pm 1 и большой полуосью $(p+1 / \rho) / 2$. Принадлежность точки $z=(\zeta+1 / \zeta) / 2$ границе области $G(n, a, \rho)$ означает равенство в левой либо правой части неравенства (3) для некоторой точки $w$. Хорошо известно, что в этом случае функция $h(w) \equiv \alpha p(\beta w ; b)$, где $\alpha$ и $\beta$ - комплексные числа, удовлетворяющие условию $|\alpha|=|\beta|=1$. Тогда из представления (2) вытекает, что $\alpha=\beta=1$ и полином $P(z)$ отображает отрезок $[-1,(d+1 / d) / 2]$ на отрезок $[-1,1]$, где $d$ из определения функции Пика. Следовательно, полином $P((z+1-b) / b)=2^{n-1} z^{n}+\cdots$ отображает отрезок $[-1,1]$ на себя (т.е. $\left.(d+1 / d) / 2=2 b^{-1}-1\right)$. Отсюда $P((z+1-b) / b) \equiv T_{n}(z)$ и $P(z) \equiv T_{n}^{a}(z)$. Характер отображения, осуществляемого полиномом $T_{n}^{a}(z)$, непосредственно виден из его определения. Теорема доказана.

ТЕОРема 2. Если полином $P(z)$ принадлежит классу $\mathscr{P}_{n}^{a}$, то для любого числа $t, 0<t<1$, образ әллипса $с$ фокусами в точках \pm 1 и большой полуосью $(t+1 / t) / 2$ при отображсении этим полиномом лежит в конечной области, ограниченной әллипсом $c$ фокусами в точках \pm 1 и большой полуосъю $(\rho+1 / \rho) / 2$, где $p\left(-\rho^{1 / n} ; b\right)=-t, b=\left(a 2^{1-n}\right)^{1 / n}$. Исключение составляет случай, когда $P(z) \equiv T_{n}^{a}(z)$ и, следовательно, все точки образа әллипса принадлежсат указанной выше области, кроме точки $-(\rho+1 / \rho) / 2$, которая лежит на ее границе.

ДокАЗАТЕЛЬСТво. Легко видеть, что для любого $t, 0<t<1$, уравнение

$$
p\left(-\rho^{1 / n} ; b\right)=-t
$$

имеет единственное решение $\rho, 0<\rho<1$. Из правого неравенства в (3) заключаем, что, если точка $z=(\zeta+1 / \zeta) / 2, \zeta=h(w)$, принадлежит эллипсу с фокусами в точ$\operatorname{kax} \pm 1$ и большой полуосью $(t+1 / t) / 2$, т.е. если $|\zeta|=t$, то соответствующая точка $w=g(\zeta)$ удовлетворяет неравенству $|w| \geqslant \rho^{1 / n}$. Это означает принадлежность точки $P(z)=\left(w^{n}+1 / w^{n}\right) / 2$ замыканию области, указанной в формулировке теоремы 2 , причем, если $P(z)$ лежит на границе этой области, то $|w|=\rho^{1 / n}$ и в правой части (3) имеет место знак равенства. В случае равенства в (3), как и при доказательстве теоремы 1 , приходим к тождеству $P(z) \equiv T_{n}^{a}(z)$. Теорема доказана.

Заметим, что при отображении полиномом $T_{n}^{a}(z), 0<a<2^{n-1}$, образ эллипса с фокусами в точках \pm 1 не является эллипсом (в отличие от случая полинома Чебьшева $T_{n}(z)$, т.е. когда $\left.a=2^{n-1}\right)$. Далее, повторяя доказательство теоремы 2 , но с привлечением левой части неравенства (3), можно получить принадлежность образа эллипса с фокусами в точках \pm 1 и большой полуосью $(t+1 / t) / 2$ "внешности” некоторого эллипca, но только в случае $t<d$. Аналог теоремы 2 , но для произвольных полиномов $P(z)$, удовлетворяющих условию (1) (без ограничения на критические значения), получен автором в работе [4, с. 26]. Доказанная там оценка также зависит от старшего коэффициента, и достигается она в случае $P(z) \equiv T_{n}(z)$. Следствие этой оценки звучит как “образ 
эллипса с фокусами в точках \pm 1 принадлежит образу этого же эллипса, но при отображении полиномом $T_{n}(z)$ " [4, следствие 3.1]. Последний результат установлен много лет назад Дочевым [6, с. 521] путем исследования соответствующих тригонометрических полиномов и впоследствии был забыт специалистами. Автору этот факт сообшил А. В. Олесов после тщательного изучения литературы.

3. Неравенства типа Маркова и Бернштейна. В работе [4, с. 41] получено неравенство типа неравенства Маркова

$$
\max \left\{\left|P^{\prime}(z)\right|: z \in[-1,1]\right\} \leqslant n^{2}\left(a 2^{1-n}\right)^{1 / n},
$$

доказательство которого полностью подходит для полиномов класса $\mathscr{P}_{n}^{a}$. Случайным образом это неравенство оказалось точным в данном классе, причем знак равенства достигается в случае $P(z) \equiv T_{n}^{a}(z)$. Соответствуюшее неравенство бернштейновского типа [4, теорема 4.5] этим свойством не обладает. Тем не менее, справедлива следующая

ТЕорема 3. Если полином $P(z)$ принадлежит классу $\mathscr{P}_{n}^{a}$, то для любого $z \in$ $[-1,1]$ выполняется неравенство

$$
\left|P^{\prime}(z) \sin \left(\frac{1}{n} \arccos P(z)\right)\right| \leqslant n\left(a 2^{1-n}\right)^{1 / n} \sqrt{1-P^{2}(z)} .
$$

Равенство в (5) для любого $z \in[-1,1]$ достигается в случае $P(z) \equiv T_{n}^{a}(z)$.

ДокАЗАТЕЛЬСТво. Позаданному полиному $P(z) \in \mathscr{P}_{n}^{a}$ построим функцию $w=g(\zeta)$ из $(2)$ и рассмотрим обратную ей функцию $\zeta=h(w)=\left(a 2^{1-n}\right)^{1 / n} w+\cdots$. Пусть

$$
\zeta_{1}=e^{i \theta}, \quad \zeta_{2}=e^{-i \theta}, \quad w_{k}=g\left(\zeta_{k}\right), \quad k=1,2, \quad 0<\theta<\pi
$$

и пусть в точках $w_{k}, k=1,2$, существуют производные $h^{\prime}\left(w_{k}\right), k=1,2$, а в точках $\zeta_{k}$, $k=1,2,-$ производные $g^{\prime}\left(\zeta_{k}\right), k=1,2$. Применяя к функции $\zeta=h(w)$ результат Поммеренке и Васильева $[7$, следствие 3.3$]$, [8, неравенство (1.1)] и вьписьвая его в терминах функции $w=g(\zeta)$, получаем

$$
\left|\frac{\zeta_{1}-\zeta_{2}}{g\left(\zeta_{1}\right)-g\left(\zeta_{2}\right)}\right| \geqslant\left(a 2^{1-n}\right)^{-1 / n}\left|g^{\prime}\left(\zeta_{1}\right) g^{\prime}\left(\zeta_{2}\right)\right|^{1 / 2} .
$$

Положим $z=\left(\zeta_{1}+1 / \zeta_{1}\right) / 2=\left(\zeta_{2}+1 / \zeta_{2}\right) / 2$. Простые вычисления дают

$$
\begin{aligned}
\left|\zeta_{1}-\zeta_{2}\right|=2 \sqrt{1-z^{2}}, \quad\left|g\left(\zeta_{1}\right)-g\left(\zeta_{2}\right)\right| & =2\left|\sin \left(\frac{1}{n} \arccos P(z)\right)\right| \\
\left|g^{\prime}\left(\zeta_{k}\right)\right| & =\frac{d|g|}{d|\zeta|}=\frac{1}{n}|\Phi(P(z))|^{1 / n-1} \frac{\left|P^{\prime}(z)\right|\left|\left(1-\zeta_{k}^{-2}\right) / 2\right|}{\sqrt{1-P^{2}(z)}} \\
& =\frac{1}{n} \frac{\left|P^{\prime}(z)\right| \sqrt{1-z^{2}}}{\sqrt{1-P^{2}(z)}}, \quad k=1,2 .
\end{aligned}
$$

Подставляя вьписанные соотношения в (6), приходим к неравенству (5) для всех точек $z$ отрезка $[-1,1]$ за исключением, может быть, конечного числа таких точек. Предельным 
переходом убеждаемся в справедливости неравенства (5) на всем отрезке $[-1,1]$. Случай равенства проверяется непосредственно. Теорема доказана.

Неравенство (5) содержит неравенство (4). Действительно, обозначим $\cos ((1 / n) \times$ $\arccos P(z))$ через $\xi$, так что $P(z)=T_{n}(\xi)$. Из неравенства $(5)$ с привлечением леммы 4.1 работы [4] имеем последовательно

$$
\begin{aligned}
\left|P^{\prime}(z)\right| & \leqslant n\left(a 2^{1-n}\right)^{1 / n} \frac{\sqrt{1-\left(T_{n}(\xi)\right)^{2}}}{\sqrt{1-\xi^{2}}} \\
& \leqslant n\left(a 2^{1-n}\right)^{1 / n} \max \left\{\sqrt{\left|T_{n}^{\prime}(\xi)\right|}: \xi \in[-1,1]\right\}=n^{2}\left(a 2^{1-n}\right)^{1 / n} .
\end{aligned}
$$

4. Неравенства для коэффициентов. Пусть, как и выше, $\zeta=h(w)$ - функция, обратная функции $(2)$, где полином $P(z) \in \mathscr{P}_{n}^{a}$. Применяя к функции $\zeta=h(w)$ известные оценки коэффициентов для регулярных и однолистных функций, приходим к некоторым неравенствам для коэффициентов полинома $P(z)$. Ограничимся следующим результатом.

ТЕОРема 4. Пусть полином

$$
P(z)=a z^{n}+\sum_{k=0}^{n-1} a_{k} z^{k}
$$

принадлежит классу $\mathscr{P}_{n}^{a}$. Тогда справедливы неравенства

$$
\begin{gathered}
n a+\left|a_{n-1}\right| \leqslant n(2 a)^{(n-1) / n}, \\
n^{2} a^{2}+\left|n^{2} a^{2}+4 n a a_{n-2}-2(n-1) a_{n-1}^{2}\right| \leqslant n^{2}(2 a)^{2(n-1) / n} .
\end{gathered}
$$

Равенство в обоих случаях достигается для полинома $T_{n}^{a}(z)$.

ДокАЗАТЕЛЬСТво. Функция

$$
w=\frac{\left(a 2^{1-n}\right)^{1 / n}}{h(1 / z)}=z+\sum_{k=0}^{\infty} A_{k} z^{-k}, \quad|z|>1
$$

принадлежит классу $\sum_{b}, b=\left(a 2^{1-n}\right)^{1 / n}$, работы Сайджека и Тамми [9]. В данной работе получены точные оценки всех коэффициентов в разложении обратной функции

$$
z=I(w)=w-\sum_{k=0}^{\infty} \beta_{k} w^{-k}, \quad|w|>1
$$

В частности, имеют место неравенства $[9$, с. 92]

$$
\left|\beta_{0}\right| \leqslant 2(1-b), \quad\left|\beta_{1}\right| \leqslant 1-b^{2} .
$$

Выписанные неравенства относятся к классическим, и они были доказаны также ранее другими авторами различными методами. Элементарные вычисления дают

$$
I(w)=\frac{1}{g(b / w)}=w+\frac{2 a_{n-1} b}{n a}+\left[1+\frac{4 a_{n-2}}{n a}-\frac{2(n-1) a_{n-1}^{2}}{n^{2} a^{2}}\right] \frac{b^{2}}{w}+\cdots,
$$


где функция $w=g(\zeta)$ задана соотношением (2). Поэтому из неравенств (7) вытекают неравенства теоремы 4 . В случае $P(z)=T_{n}^{a}(z)$ функция $\zeta=h(w)$ является функцией Пика, для которой в неравенствах (7), как известно, достигается знак равенства. Этим завершается доказательство теоремы 4.

Учитывая полученные здесь результаты, можно предположить, что полином $T_{n}^{a}(z)$ является экстремальным также в аналогичных задачах для более широкого класса полиномов (без ограничения на критические значения).

\section{СПИСОК ЦИТИРОВАННОЙ ЛИТЕРАТУРЫ}

[1] Milovanovic G.V., Mitrinovic D.S., Rassias Th. M. Topics in Polynomials: Extremal Problems, Inequalities, Zeros. Singapore: World Scientific Publ., 1994.

[2] Borwein P., Erdelyi T. Polynomials and Polynomial Inequalities. Grad. Texts in Math. V. 161. New York: Springer-Verlag, 1995.

[3] Govil N. K., Mahapatra R. N. Markov and Bernstein type inequalities for polynomials // J. Inequal. Appl. 1999. V. 3. P. 349-387.

[4] Дубинин В.Н. Конформные отображения и неравенства для алгебраических полиномов // Алгебра и анализ. 2001. Т. 13. № 5. С. 16-43.

[5] Goodman A. W. Univalent Functions. I. II. Tampa: Mariner Publ. Comp., 1983.

[6] Дочев К. О некоторых экстремальных свойствах многочленов // Докл. АН СССР. 1963. T. 153. № 3. C. 519-521.

[7] Pommerenke Ch., Vasil'ev A. On bounded univalent functions and the angular derivative // Ann. Univ. Maria Curie-Sklodowska. Ser. A. 2000. V. 8. P. 79-106.

[8] Pommerenke Ch., Vasil'ev A. Angular derivatives of bounded univalent functions and extremal partitions of the unit disk // Pacific J. Math. 2002. V. 206. № 2. P. 425-450.

[9] Siejka H., Tammi O. On estimating the inverse coefficients for meromorphic univalent functions omitting a disc // Ann. Acad. Sci. Fenn. Ser. A. I. Math. 1987. V. 12. P. 85-93.

Институт прикладной математики ДВО РАН, г. Владивосток

Поступило

E-mail: dubinin@iam.dvo.ru

25.02 .2005 